\title{
Ethnomedicinal appraisal and conservation status of medicinal plants among the Manobo tribe of Bayugan City, Philippines
}

\author{
MARK LLOYD G. DAPAR ${ }^{1,3, v}$, ULRICH MEVE ${ }^{3}$, SIGRID LIEDE-SCHUMANN ${ }^{3}$, \\ GRECEBIO JONATHAN D. ALEJANDRO ${ }^{1,2,3}$ \\ ${ }^{1}$ Graduate School and Research Center for the Natural and Applied Sciences, University of Santo Tomas. España Boulevard, 1015 Manila, Philippines. \\ Tel.: +63-2-34061611, ^email: marklloyd.dapar.gs@ust.edu.ph \\ ${ }^{2}$ College of Science, University of Santo Tomas. España Boulevard, 1015 Manila, Philippines \\ ${ }^{3}$ Department of Plant Systematics, University of Bayreuth. Universitätsstr. 30, D-95440 Bayreuth, Germany
}

Manuscript received: 10 July 2020. Revision accepted: 28 July 2020.

\begin{abstract}
Dapar MLG, Meve U, Liede-Schumann S, Alejandro GJD. 2020. Ethnomedicinal appraisal and conservation status of medicinal plants among the Manobo tribe of Bayugan City, Philippines. Biodiversitas 21: 3843-3855. Manobo tribe is one of the most populated indigenous communities in the Philippines clustered in various parts of Mindanao archipelago with distinct cultural traditions and medicinal practices. This study aims to document the Agusan Manobo tribe medicinal plant uses and knowledge and to assess the conservation status of their medicinal plants found in upland ancestral lands where ethnomedicinal practices still prevail. Ethnomedicinal data were gathered from 95 key informants through semi-structured interviews, focus group discussions, and guided field walks in five selected upland barangays of Bayugan City. Family importance value (FIV) and relative frequency of citation (RFC) were quantified. The conservation status of their medicinal plants was assessed based on the international and national listing of threatened species. A total of 90 species belonging to 82 genera and 41 families were identified as ethnomedicinally important. Highest FIV (98.9) was reported for Piperaceae in treatment for skin diseases and infections. The most cited medicinal plant species was Piper decumanum $\mathrm{L}$. ( $\mathrm{RFC}=0.95$ ), which is primarily used in treatment for insect and snake bites. Conservation assessment revealed that the five upland areas of the Agusan Manobo tribe are habitats of endemic, endangered, vulnerable and threatened species. The results of this study present the rich ethnomedicinal knowledge of Agusan Manobo cultural community, which could serve as a useful source of information to improve community healthcare and environmental conservation and management. Local people and the government should actively participate in shared management responsibilities for viable conservation strategies and sustainable use of the cultural community resources.
\end{abstract}

Keywords: Ethnobotany, Manobo tribe, medicinal plants, Mindanao, Philippines

\section{INTRODUCTION}

Recent biodiversity global assessment reported around one million animal and plant species (SDG 2019), and more than 28,000 species (IUCN 2019) are now threatened with accelerating extinction rate more than ever before in human history. Given this emerging biodiversity crisis, ancestral lands governed by indigenous communities are significantly declining at a slower rate (IPBES 2019). The rich knowledge, ecological understanding, resource management, and conservation practices of the locals and indigenous peoples are recognized as imperative partners in environmental management because they act as stewards of their ancestral territories (UNESCO 2019). Indigenous and local knowledge (ILK) is accepted among researchers and policymakers as essential for biodiversity conservation worldwide (Reyes-García and Benyei 2019). Indigenous communities have long experienced, coped, and adjusted to environmental changes over the years (Vinyeta and Lynn 2013). Considering the experience of indigenous people, their knowledge must play an integral role in sustaining global biodiversity and protect world heritage.

An estimated $22 \%$ of the world's land surface was acknowledged as indigenous ancestral lands, which correspond to about $80 \%$ of the plant's biodiversity areas (WRI 2005). Despite the indigenous transformation of various cultural knowledge systems and traditional practices in sustaining forest reserves, biodiversity has prevailed in the Philippines (Camacho et al. 2015). The Philippine archipelago is comprised of more than 7,100 islands and islets. This country is considered significantly crucial to global biodiversity due to its exceptional levels of narrow endemism in various ecosystems (Myers et al. 2000; Carpenter and Springer 2005; Posa et al. 2008). Aside from being renowned as a megadiverse country worldwide (Conservation International 2012) and one of the world's eight biodiversity hottest hotspots (Myers et al. 2000), the Philippines is also recognized as culturally megadiverse in ethnicity accounting for 110 divergent ethnolinguistic groups (ILO 2014, PSA 2016). These various indigenous communities have a prominent and unique identity, language, and cultural practices (NCIP 2010).

Mindanao is mostly occupied with $61 \%$ of the total number of indigenous peoples (IPs) in the Philippines (UNDP 2010). One of the largest groups of IPs in Mindanao is the Manobo tribe, mainly inhabiting the province of Agusan del Sur known as the Agusan Manobo 
(NCIP 2010; NCCA 2015; Reyes et al. 2017). Etymologically, the Manobo term was named after the "Mansuba," which means river people, coined from the "man" (people) and the "suba" (river). Like other indigenous communities in the country, Agusan Manobo occupied their ancestral lands situated in uplands and hinterlands with harmony for generation, protected their natural resources, and maintained the integrity of their ecosystem (Dapar et al. 2020a). The rich traditional knowledge of indigenous and local people with regards to biodiversity resources is continually supported by the Intergovernmental Platform on Biodiversity and Ecosystem Services (IPBES 2019) and the Convention on Biological Diversity (CBD 2019) of the United Nations. Their critical roles as conservation actors are essential for joint intervention with Indigenous Peoples Organization (IPOs) to strategize biodiversity programs around the globe (Alcorn 2010).

Despite the significant role of indigenous contributions to biodiversity conservation, limited studies have so far been conducted in the Philippines among Indigenous Cultural Communities/Indigenous Peoples (ICCs/IPs) in documenting relative importance and conservation status of their medicinal plants. Bayugan City, the only component city in the province of Agusan del Sur and known to be occupied with the large number of Agusan Manobo who serve as stewards of their abundant natural resources, remains underdocumented. Hence, it is paramount to evaluate plant resources like medicinal plants among indigenous communities and check for their conservation status as baseline information and justification for future conservation. Thus, this study aims to document ethnomedicinal importance and to assess the conservation status of the medicinal plants used by the Agusan Manobo in upland areas of Bayugan City, Agusan del Sur, Philippines.

\section{MATERIALS AND METHODS}

\section{Study area}

The fieldwork was undertaken in the City of Bayugan as the only component city in the province of Agusan del Sur, Philippines, as shown in Figure 1. This landlocked city is located in the coordinates $8^{\circ} 71^{\prime} 44^{\prime \prime} \mathrm{N}, 125^{\circ} 74^{\prime} 81^{\prime \prime} \mathrm{E}$ with type II climate having no dry season but very pronounced wet season with heavy precipitation. It is geographically situated below the typhoon belt near or alongside the eastern coast of Mindanao, which is typically affected by tropical depression and typhoon passing the province of Surigao del Norte and Visayas regions. From the total of 43 barangays of the city, five upland barangays, namely Mt. Ararat (523.7 masl), Mt. Carmel (360.8 masl), Mt. Olive (323.2 masl), New Salem (628.5 masl), and Pinagalaan (523.1 masl), were purposively selected for the reasons of availability, accessibility, and security with the approved consent of the tribal council, provincial administration of Agusan del Sur, the National Commission on Indigenous Peoples (NCIP) of the LGU and CARAGA Administrative Region, and the Provincial Environment and Natural Resources Office (PENRO) of Agusan del Sur, and the Department of Environment and Natural Resources (DENR) of CARAGA. These five study sites partly comprised the $74 \%$ forestland areas and $26 \%$ alienable and disposable (A\&D) areas of the province (PENRO Agusan del Sur 2019). There were three hospitals in the downtown city, which is distant from the five remote barangays of the study, therefore, with poor access to the city healthcare.

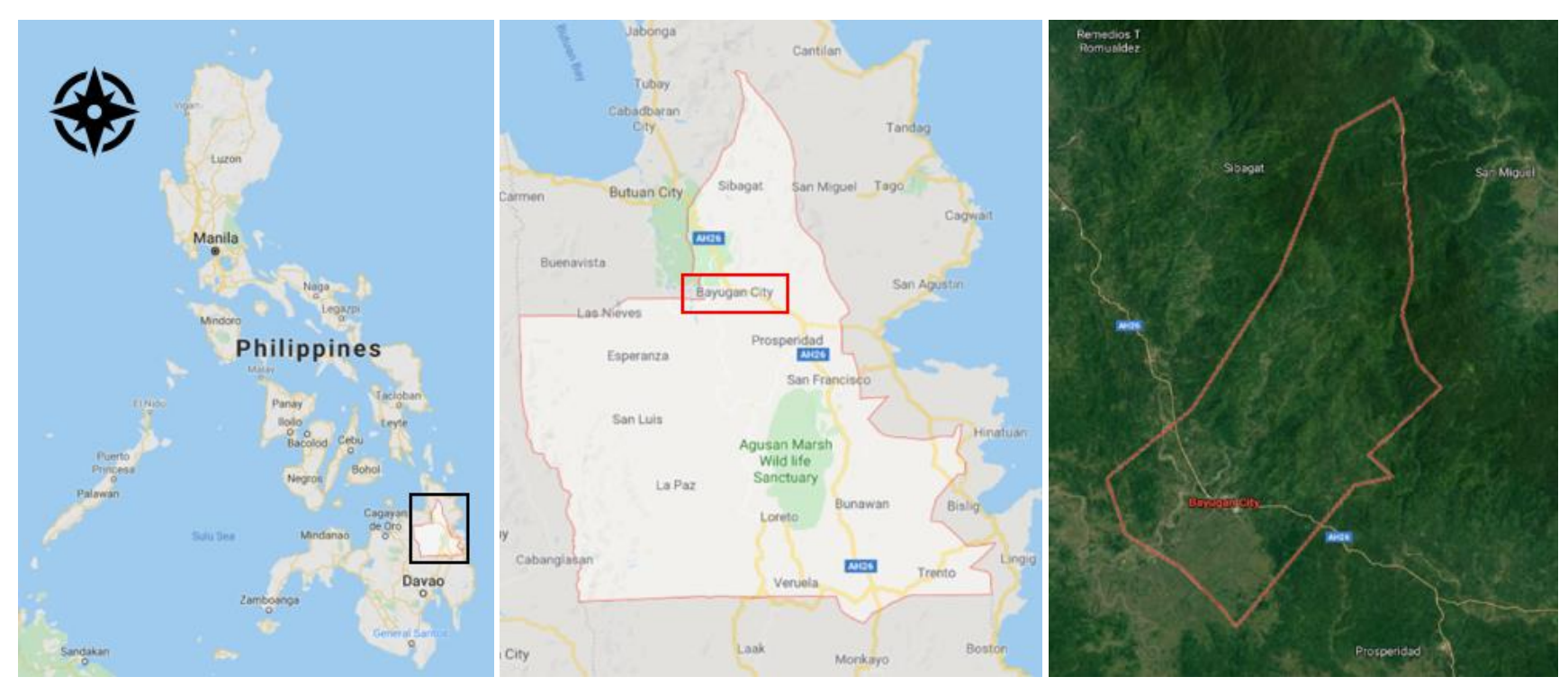

Figure 1. Location map of Bayugan City (C), Agusan del Sur (A, B), Philippines (Maps by Google Earth) 


\section{Field survey}

Fieldwork was carried out from March 2018 to April 2019. The work consisted of the acquisition of ethics approval, consents, resolution, certification, and permit before the actual interview, field survey, and collections in the selected five upland barangays. Consultative assembly was conducted with the tribal council of elders in cooperation with the city administration to talk about the research intent as wholly academic. After consultations and meetings, the fieldwork was followed by ritual observation resulting in mutual agreement and respect. As approved by the council of elders, the survey and sampling were certified by the NCIP-LGU and NCIP-CARAGA following their by-laws for the welfare and protection of IPs.

A total of 95 purposively and snowball sampled AgusanManobo key informants, more than $10 \%$ of the total population of selected barangays, comprising of the tribal council of elders and members, were interviewed using the semi-structured open-ended interview. Key informants were composed of 39 females and 56 males with an age range from 18 to 77 years old and the median age is 38 . Ethnomedicinal data were consolidated from the interviews and corner meetings with the key informants. An accurate translation to the Manobo dialect, known as Minanubu, with the help of the tribal elders, was secured. Focus group discussions (FGD) among respondents have jointly participated with the respective barangay tribal leaders and the only tribal healer and in cooperation with the city Indigenous Peoples Mandatory Representative (IPMR) as a consultant. FGD is helpful to clarify the main points, ideas, and perceptions on the use of traditional folk medicines, awareness about the conservation of their medicinal plant cultural resources and indigenous knowledge.

\section{Collection and identification}

All collected medicinal plants during field walks and sampling were carefully pressed, alcohol-preserved, dried, and mounted on herbarium sheets. Voucher specimens were deposited in the University of Santo Tomas Herbarium (USTH). Recorded vernacular names were compared to the Dictionary of Philippines Plant Names by Madulid (2001). Plant identification was verified by Mr. Danilo Tandang, a botanist and researcher at the National Museum of the Philippines. All scientific names were checked for spelling and synonyms, and family classification using The Plant List (2013), World Flora Online (2019), the International Plant Names Index (2019), and Tropicos (2019). Medicinal plant species occurrence, distribution, and species identification were further confirmed in the updated Co's Digital Flora of the Philippines (CDFP; Pelser et al. 2011 onwards).

\section{Family importance value (FIV)}

FIV determines the local importance of the families of medicinal plant species (Ali et al. 2018). This value is calculated based on the number of informants citing the family using the formula: FIV $=(\mathrm{FC} / \mathrm{N}) \times 100$, where FC is the frequency of citation of the plant family, and $\mathrm{N}$ is the total number of informants. FIVranges in value from 0 to 100 , with the most important family having values closer to 100.FIV distinguishes the most important family based on the number of citation reports of informants and the number of medicinal plant species for treatment.

\section{Relative frequency of citation (RFC)}

RFC identifies the local importance of each medicinal plant species (Ugulu et al. 2009). This index is calculated using this formula: $\mathrm{RFC}=\mathrm{FC} / \mathrm{N}$, where $\mathrm{FC}$ (frequency of citation) is the number of informants who stated the medicinal plant, and $\mathrm{N}$ is the total number of informants. FC assigned the most preferred or more used medicinal plant species. At the same time, RFC determines the traditional importance of plant species in the study site ranging in value from 0 to 1 , with the most critical species having values closer to 1 .

\section{Conservation status and endemicity}

Conservation status of the medicinal plants was assessed based on the international data of the IUCN (2019) updated national list of threatened Philippine plants and their categories of the DENR Administrative Order No. (DAO) 2017-01 (DENR Administrative Order 2017) and the consolidated national online flora database of Co's Digital Flora of the Philippines (CDFP; Pelser et al. 2011 onwards). The collected medicinal plants were further checked for their occurrence and distribution in the Philippines and to check their endemicity.

\section{RESULTS AND DISCUSSION}

\section{Characteristics of medicinal plants}

During the ethnobotanical survey of medicinal plants, a total of 90 species belonging to 82 genera and 41 families were identified and recorded with medicinal uses, as shown in Table 1. Most of the documented medicinal plants were trees $(35 \%)$, followed by herbs $(33 \%)$, shrubs $(20 \%)$, and climbers $(12 \%)$, as depicted in Figure 2. Several plant parts are used by Agusan Manobo for various health problems, as shown in Figure 3. The most medicinally most used plant parts are the leaves $(39 \%)$, followed by roots $(22 \%)$, stems and barks (12\%), while the least used ones are rhizomes (3\%), shoots $(2 \%)$, fruits $(2 \%)$, flowers $(2 \%)$, and branches $(1 \%)$.

\section{Family importance value}

Analysis of FIV revealed that Piperaceae has the highest value (98.9), followed by Lauraceae (96.8), Apocynaceae (94.7), and Euphorbiaceae (90.5). These families were highly cited by the informants as frequently used for treatment against skin diseases and infections, cough, wounds, and fever, respectively.

\section{Relative frequency of citation}

The highest RFC values were recorded for Piper decumanum L. (0.95), followed by Anodendron borneense (King \& Gamble) D.J.Middleton (0.89), Micromelum minutum (G.Forst.) Wight \& Arn. (0.81), Arcangelisia flava (L.) Merr. (0.81), and Cinnamomum mercadoi S.Vidal (0.79). These medicinal plants are highly cited as a treatment for insect and snake bites, pregnancy problems (impotence and sterility), cancer, ulcer, and diarrhea, respectively. 
Table 1. List of documented medicinal plants with their conservation status, endemicity, medicinal parts, and uses (alphabetically arranged by family)

\begin{tabular}{|c|c|c|c|c|c|c|c|c|}
\hline \multirow{2}{*}{ Scientific name } & \multirow[t]{2}{*}{ Voucher No. } & \multirow{2}{*}{ FIV } & \multirow{2}{*}{ RFC } & \multicolumn{2}{|c|}{$\begin{array}{c}\text { Conservation } \\
\text { status }\end{array}$} & \multirow{2}{*}{$\begin{array}{l}\text { Endemicity } \\
(\text { CDFP })^{\mathrm{c}}\end{array}$} & \multirow{2}{*}{$\begin{array}{l}\text { Parts } \\
\text { used }^{\text {d }}\end{array}$} & \multirow{2}{*}{ Medicinal uses } \\
\hline & & & & $\overline{\text { IUCN }^{\mathbf{a}}}$ & $\begin{array}{l}\text { DENR/ } \\
\text { CDFP }^{b}\end{array}$ & & & \\
\hline Acanthaceae & & 68.42 & & & & & & \\
\hline Rhinacanthus nasutus (L.) Kurz & USTH 015622 & & 0.52 & NA & & $\mathrm{NE}$ & Lf & Colds, beriberi, diabetes, diarrhea, fatigue, spasm \\
\hline Anacardiaceae & & 63.16 & & & & & & \\
\hline Mangifera indica $\mathrm{L}$. & USTH 015591 & & 0.20 & $\mathrm{DD}$ & & $\mathrm{NE}$ & $\mathrm{Fr}, \mathrm{Lf}$ & Constipation, cough, diarrhea, stomach trouble \\
\hline Spondia spinnata (L.f.) Kurz & USTH 015599 & & 0.33 & NA & & $\mathrm{NE}$ & $\mathrm{Bk}, \mathrm{Lf}$ & Colds, cough, diabetes, fever \\
\hline Annonaceae & & 36.84 & & & & & & \\
\hline Friesodielsia lanceolata (Merr.) Steen. & USTH 015558 & & 0.18 & NA & & EN & $\mathrm{Bk}, \mathrm{Lf}, \mathrm{Rt}$ & t Postpartum care and recovery \\
\hline Uvaria zschokkei Elmer & USTH 015662 & & 0.17 & NA & & EN & $\mathrm{St}$ & Amoebiasis, hypertension, fatigue \\
\hline Apocynaceae & & 94.74 & & & & & & \\
\hline Alstonia macrophylla Wall. ex G.Don & USTH 015546 & & 0.35 & $\mathrm{LC}$ & & $\mathrm{NE}$ & $\mathrm{Bk}$ & Animal bites, skin diseases and infections, wounds \\
\hline $\begin{array}{l}\text { Anodendron borneense (King \& Gamble) } \\
\text { D.J.Middleton }\end{array}$ & USTH 015639 & & 0.89 & NA & & $\mathrm{NE}$ & $\mathrm{St}$ & $\begin{array}{l}\text { Arthritis, body pain, cancer, diabetes, fatigue, pregnancy } \\
\text { (impotence and sterility), skin diseases, spasm, wounds }\end{array}$ \\
\hline Araceae & & 9.47 & & & & & & \\
\hline Alocasia zebrina Schott ex Van Houtte & USTH 015614 & & 0.05 & NA & VU & EN & St & Snake bites and wounds \\
\hline $\begin{array}{l}\text { Homalomena philippinensis Engl. ex Engl. } \\
\text { \&K.Krause }\end{array}$ & USTH 015597 & & 0.04 & NA & & EN & $\mathrm{Rz}$ & Labor and delivery enhancer, rheumatism, wounds \\
\hline Araliaceae & & 29.47 & & & & & & \\
\hline Hydrocotyle vulgaris $\mathrm{L}$. & USTH 015563 & & 0.23 & $\mathrm{LC}$ & & $\mathrm{NE}$ & Lf & Diabetes, hypertension \\
\hline Arecaceae & & 22.11 & & & & & & \\
\hline Calamus megaphyllus Becc. & USTH 015608 & & 0.15 & NA & NT & EN & $\mathrm{Rz}$ & Arthritis, asthma, diarrhea, hypertension \\
\hline Asteraceae & & 88.42 & & & & & & \\
\hline Acmella grandiflora (Turcz.) R.K.Jansen & USTH 015548 & & 0.35 & NA & & $\mathrm{NE}$ & Fw & Skin rashes and itchiness, toothache \\
\hline Artemisia vulgaris $\mathrm{L}$. & USTH 015619 & & 0.33 & $\mathrm{LC}$ & & $\mathrm{NE}$ & $\mathrm{Lf}$ & Asthma, body pain, cough, fever, spasm \\
\hline Bidens pilosa $\mathrm{L}$. & USTH 015582 & & 0.20 & NA & & $\mathrm{NE}$ & Rt & Body pain, diarrhea, fatigue, gas pain and flatulence \\
\hline Blumea balsamifera (L.) DC. & USTH 015573 & & 0.37 & LC & $\mathrm{LC}$ & $\mathrm{NE}$ & Lf & Cough, fatigue, fever, headache, hypertension, spasm \\
\hline Chromolaena odorata (L.) R.M.King\&H.Rob. & USTH 015632 & & 0.40 & NA & & $\mathrm{NE}$ & Lf & Burns, fever, skin diseases, wounds \\
\hline Cyanthillium cinereum (L.) H.Rob. & USTH 015587 & & 0.43 & NA & & $\mathrm{NE}$ & Lf, Rt & $\begin{array}{l}\text { Animal bites, colds, malaria, measles, skin diseases, spasm, } \\
\text { tuberculosis }\end{array}$ \\
\hline $\begin{array}{l}\text { Erechtites valeriani folius (Link ex } \\
\text { Spreng.) DC. }\end{array}$ & USTH 015666 & & 0.19 & NA & & $\mathrm{NE}$ & $\mathrm{Lf}$ & Gas pain and flatulence, stomach trouble, wounds \\
\hline Mikania cordata (Burm.f.) B.L.Rob. & USTH 015543 & & 0.36 & NA & & $\mathrm{NE}$ & $\mathrm{Lf}$ & $\begin{array}{l}\text { Animal and insect bites, skin diseases and infections, ulcer, } \\
\text { wounds }\end{array}$ \\
\hline Pseudelephantopus spicatus (Juss.) Rohr & USTH 015564 & & 0.45 & NA & & $\mathrm{NE}$ & Lf, Rt & $\begin{array}{l}\text { Kidney problem, menstrual problem, skin diseases, } \\
\text { snakebite, wounds }\end{array}$ \\
\hline
\end{tabular}


Athyriaceae

Diplazium esculentum (Retz.) Sw.

Boraginaceae

Ehretia microphylla Lam.

Byttneriaceae

Abroma augusta (L.) L.f.

Melochia umbellata (Houtt.) Stapf Campanulaceae

Hippobroma longiflora (L.) G.Don Caricaceae

Carica papaya $\mathrm{L}$.

Costaceae

Hellenia speciosa (J.Koenig) Govaerts

Cyperaceae

Rhynchospora colorata (L.) H.Pfeiff.

Dioscoreaceae

Stenomeris borneensis Oliv.

Euphorbiaceae

Euphorbia hirta L.

Jatropha curcas L.

Jatropha gossypifolia L.

Melanolepis multiglandulosa (Reinw. ex Blume) Rchb. \& Zoll.

Omalanthus macradenius Pax \& Hoffm.

Fabaceae

Bauhinia cf. semibifida

Mimosa pudica $\mathrm{L}$

Ormosia macrodisca Baker

Phanera semibifida (Roxb.) Benth.
25.26

USTH 015545

USTH 015638

USTH 015637

36.8

$0.30 \quad \mathrm{NA}$

$0.29 \quad \mathrm{NA}$

USTH 015649

USTH 015583

USTH 015668

USTH 015578

USTH 015571

USTH 015537 58.95

$0.48 \quad$ NA

90.53

USTH 015665

USTH 015595

USTH 015586

USTH 015621

USTH 015633

USTH 015575

USTH 015570

USTH 015625

USTH 015646

$0.27 \quad$ NA

$0.44 \quad$ NA

0.73 LC

0.44 LC

Sh

Lf

Allergy, diabetes, hyperacidity

Bk, Lf, Rt Diabetes, menstrual problem, skin diseases and infection, stomach problem, wounds

Lf Body pain, burns, canker sore, rheumatism

Fr Ascariasis, cancer, constipation

Lf Body pain, constipation, dengue fever

$\mathrm{NE}$

Lf, St, Rz Cough, diabetes, fever, goiter, kidney problem, stomach trouble

Wh

Chickenpox, gas pain and flatulence, hyperacidity, measles

$\mathrm{NE}$

Rt

Arthritis, migraine, myoma, rheumatism, spasm, urinary problem

Wh Colds, dengue fever, fever, skin diseases

Lf, Rt Arthritis, body pain, diarrhea, fatigue, fever, menstrual problem, spasm, tuberculosis

Lf, Rt Body pain, diarrhea, dysmenorrhea, fatigue, fever, menstrual problem, tuberculosis

Lf Beriberi, diarrhea, emphysema, fatigue

Skin diseases and infections, wounds

Lf, St Body pain, postpartum care and recovery, rheumatism, spasm

Rt Child sleeplessness, diabetes, dysentery, hypertension, urinary problem

NE Bk, Rt High cholesterol, kidney problem, nervous breakdown,

NE typhoid fever

Lf Hemorrhage, internal bleeding 
Lamiaceae

Callicarpa pedunculata R.Br.

Coleus amboinicus Lour.

Coleus scutellarioide s(L.) Benth.

Gmelina arborea Roxb. ex Sm

Hyptis capitata Jacq.

Ocimum basilicum $\mathrm{L}$.

Orthosiphon aristatus (Blume) Miq.

Premna odorata Blanco

Teijsmanniodendron ahernianum (Merr.) USTH 015603 Bakh.

Vitex negundo L.

Lauraceae

Cinnamomum mercadoi S.Vidal

Litsea cordata (Jack) Hook f.

Machilus philippinensis Merr.

Lythraceae

Lagerstroemia speciosa (L.) Pers.

Malvaceae

Gossypium hirsutum L.

Sidar rhombifolia $\mathrm{L}$.

Urena lobata $\mathrm{L}$

Marattiaceae

Angiopteris evecta $\mathrm{Sw}$

Melastomataceae

Medinilla teysmannii Miq.

Melastoma malabathricum $\mathrm{L}$.
USTH 015562

USTH 015585

USTH 015580

USTH 015576

USTH 015596

USTH 015553

USTH 015601

USTH 015664

84.21

96.84

34.74

15.79

USTH 015658

USTH 015581

USTH 015588

$\begin{array}{ll}0.34 & \mathrm{~N} \\ 0.34 & \mathrm{~N} \\ 0.37 & \mathrm{~N}\end{array}$

0.30 LC

$0.45 \quad$ NA

$0.35 \quad \mathrm{NA}$

$0.46 \quad \mathrm{NA}$

$0.60 \quad \mathrm{NA}$

LC

0.11 LC

$0.43 \quad$ NA

0.07

0.25

0.69

0.43

NA

LC

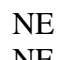

$\mathrm{NE}$

$\mathrm{NE}$

NE

$\mathrm{NE}$

NE

NE

EN

NE

NE

OTS

EN

NE

OTS

$\mathrm{NE}$

NE

NE

NE

NE

0.11

0.25 NA

OTS

NE

NE
Lf Asthma, body pain, fatigue, muscle pain, spasm, ulcer

Lf Abdominal pain, asthma, cough, gas pain and flatulence

Lf Anemia, asthma, cough, gas pain and flatulence, pneumonia, Anemia, asthma, cough, gas pain and flatulence, pneumonia,
tuberculosis, ulcer tuberculosis, ulcer

Lf Gas pain and flatulence, maternal care, stomach bloating, wounds

Lf Diarrhea, gas pain and flatulence, new-born baby care, wounds $\mathrm{Sh}$

Constipation, cough, diarrhea, menstrual problem,

postpartum care and recovery

Fw, Lf Arthritis, diabetes, diarrhea, hypertension, kidney problem, prostate problem, urinary problem

Lf Colds, cough, diarrhea, fatigue, postpartum care and

recovery, rheumatism, wounds

Rt, St Beriberi, body pain, labor and delivery enhancer, muscle pain, spasm

Lf Cough, gas pain and flatulence, headache, postpartum care and recovery

$\mathrm{Bk}, \mathrm{Br}$ Amoebiasis, cancer, cough, diarrhea, fatigue, hyperacidity, Rt kidney problem, spasm, urinary problem

Bk, Rt Asthma, cough, goiter, myoma, pneumonia, tumor

St Cough, fatigue

Lf Body pain, fever, kidney problem, ulcer, urinary problem

Rt Body pain, chilling, fever, hemorrhage, postpartum care and recovery

Lf, Rt Body pain, chickenpox, cough, kidney, menstrual, prostate and stomach problems, skin infection, wounds

Wh Animal bites, arthritis, bruises, fracture and dislocation, labor and delivery enhancer, postpartum care and recovery,

rheumatism

$\mathrm{Rt}$

Cramp and spasm, postpartum care and recovery

Lf

Sh
Gas pain and flatulence, muscle pain, sprain and swellings

Diarrhea, dysentery, fever, headache, hemorrhoids 
Meliaceae

Sandoricum koetjape (Burm.f.) Merr. $\quad$ USTH 015624

Swietenia mahagoni (L.) Jacq. $\quad$ USTH 015671

Menispermaceae

Arcangelisia flava (L.) Merr.

USTH 015600

Tinospora crispa (L.) Hook.f. \& Thomson USTH 015566

Moraceae

Ficus concinna (Miq.) Miq.

USTH 015552

Ficus fistulosa Reinw. ex Blume

USTH 015561

Ficus pseudopalma Blanco

USTH 015636

Ficus septic Burm.f

USTH 015623

Morus alba $\mathrm{L}$.

Muntingiaceae

Muntingia calabura $\mathrm{L}$

Myristicaceae

Myristica agusanensis Elmer

Pandanaceae

Pandanus amaryllifolius Roxb.

Phyllantaceae

Phyllanthus amarus Schumach. \&Thonn.

USTH 015549

USTH 015629

28.42

USTH 015611

USTH 015555

USTH 015590

Piperaceae

Piper aduncum L.

Piper decumanum $\mathrm{L}$.

USTH 015568

USTH 015544

Pipercf. nigrum, 'wild'

USTH 015560

Poaceae

Eleusine indica (L.) Gaertn.

Imperata cylindrica (L.) P.Beauv.

USTH 015569

57.89

USTH 015605

USTH 015627

47.37

83.16

23.16

98.95

Paspalum conjugatum P.J.Bergius
$0.15 \quad$ NA

$0.81 \quad$ NA

$0.70 \quad \mathrm{NA}$

$0.54 \quad$ LC

0.43 LC

$0.30 \quad$ NA

0.52 LC

$0.17 \quad \mathrm{VU}$

$0.18 \quad \mathrm{NA}$

$0.41 \quad$ NA

$0.26 \quad$ LC

0.95 NA

0.74

Lf
Bk, Lf Abdominal pain, diarrhea, skin diseases and infections

Bk Amoebiasis, cough, diarrhea, fever, hypertension

Rt, St Diabetes, diarrhea, jaundice, menstrual problem, myoma, skin diseases and infections, tumor, ulcer

St Arthritis, body pain, diarrhea, malaria, menstrual problem,

rheumatism, stomach trouble, ulcer, wounds

Bk, Lf, Rt Arthritis, cancer, cyst, fracture and dislocation, kidney and prostate problems, tumor, wounds

Bk, Rt Asthma, colds, cough, diabetes, fatigue, hypertension,

postpartum care and recovery, respiratory problem

Lf, Rt Diabetes, hemorrhage, hypertension, kidney problem,

postpartum care and recovery, spasm, stomach trouble

Lf, Rt Asthma, body pain, cataract, fatigue, fever, herpes simplex, muscle pain, skin diseases and infections, stomach trouble,

warts

Lf Asthma, colds, cough, muscle pain, pneumonia, spasm

Abdominal pain, colds, diarrhea, headache, stomach trouble, ulcer

\section{Bk Measles, bronchitis and other respiratory diseases}

Heart, kidney, prostate and urinary problems

Wh Colds, fever, jaundice, kidney, new-born baby care, stomach and urinary problems

Asthma, cough, fracture and dislocation

Arthritis, body pain, cancer, cyst, diarrhea, poisoning, skin diseases and infections, tuberculosis, snake and insect bites, tumor, ulcer, wounds

Acne, animal bites, body pain, cancer, cyst, diarrhea, gas pain and flatulence, poisoning, skin diseases and infections, tumor, wounds

Arthritis, diabetes, diarrhea, internal bleeding, kidney problem, postpartum care and recovery, spasm

Chickenpox, diarrhea, fever, measles, toothache, urinary

problem,

Rt Diarrhea, dysentery, hair loss, wounds 
Rubiaceae

Uncaria lanosa Wall.

Rutaceae

Melicope latifolia (DC.) T.G.Hartley USTH 015540

Melicope cf. triphylla

Arn.

Solanacea

Capsicum annuum $\mathrm{L}$.

Sparmanniaceae

Grewia laevigata Vah

USTH 015626

USTH 015547

Urticaceae

Dendrocnide luzonensis (Wedd.) Chew

Oreocnid erubescens (Blume) Miq.

Pipturus arborescens (Link) C.B.Rob.

USTH 015572

USTH 015676

USTH 015673

Poikilospermum acuminatum (Trecul.)

Merr.

Verbenaceae

Stachytarpheta jamaicensis (L.) Vah

Xanthorrhoeaceae

Dianella ensifolia (L.) DC.

USTH 015655

USTH 015594

USTH 015656

Zingerberaceae

Curcuma longa $\mathrm{L}$

USTH 015674
13.68

85.26

$0.04 \quad$ NA

0.08

$0.81 \quad$ NA

St

Diarrhea, stomach trouble

Cough

St Carbuncle

Lf, St, Rt Arthritis, animal and insect bites, body pain, cancer, rheumatism, skin diseases and infections, swellings, ulcer, wounds

13.68

44.21

\section{$0.14 \quad$ LC}

NE

Lf Appetite enhancer, insect bites, skin diseases and infections

$0.42 \quad$ LC

$\mathrm{NE}$

Bk, Lf, Rt Body pain, diabetes, fatigue, fever, hypertension, labor and delivery enhancer, relapse, spasm

64.21

0.08 NA EN Rt Constipation, cough, fatigue, fever, hormonal imbalance,

$0.14 \quad$ LC

$0.48 \quad$ NA

NE

$\mathrm{NE}$

Lf Cough, fever, stomach trouble

Bk, Lf Anxiety and depression, body pain, fatigue, hyperacidity, skin diseases and infections, wounds

0.22 NA NE $\quad$ St, Rt Animal and insect bites, postpartum care and recovery, skin diseases and infections, sore eyes, ulcer

41.05

25.26

$0.35 \quad \mathrm{NA}$

NE

Lf, Rt Ascariasis, boils, bruises, fever, sprain

$0.15 \quad$ NA

NE

Lf, Rt Maternal care, milk production enhancer, postpartum care and recovery

34.74

Note: aUCN: NA: Not Assessed, DD: Data Deficient, LC: Least Concern, VU: Vulnerable, EN: Endangered. 'bENR Administrative Order 2017-11, CDFP: LC: Least Concern, NT: Near Threatened, OTS: Other Threatened Species. ${ }^{\mathrm{C} C D F P}$ : EN: Endemic, NE: Not Endemic. ${ }^{\mathrm{d}}$ Bk: barks; Br: branches; Fr: fruits; Fw: flowers; Lf: leaves; Rt: roots; Rz: rhizomes; Sh: shoots; St: stems; Wh: whole plants. 


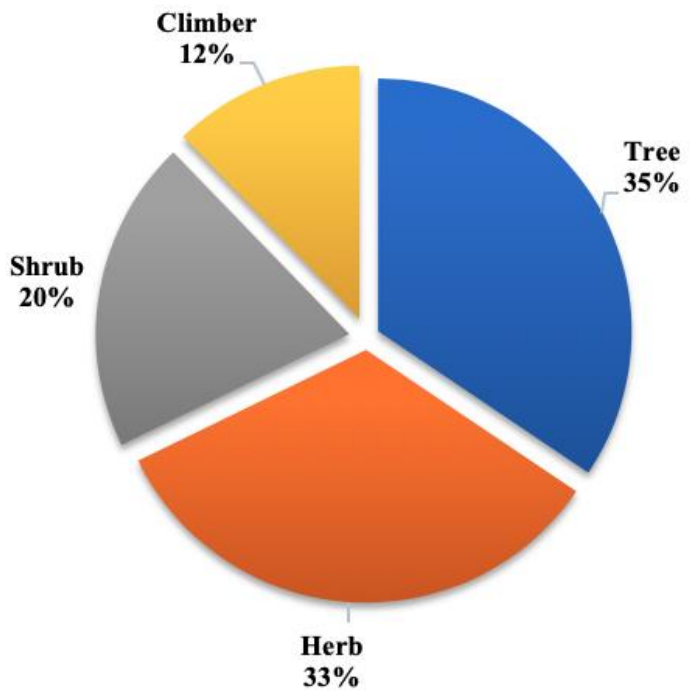

Figure 2. Habit of medicinal plants collected from the five upland barangays of Bayugan City, Agusan del Sur, Philippines

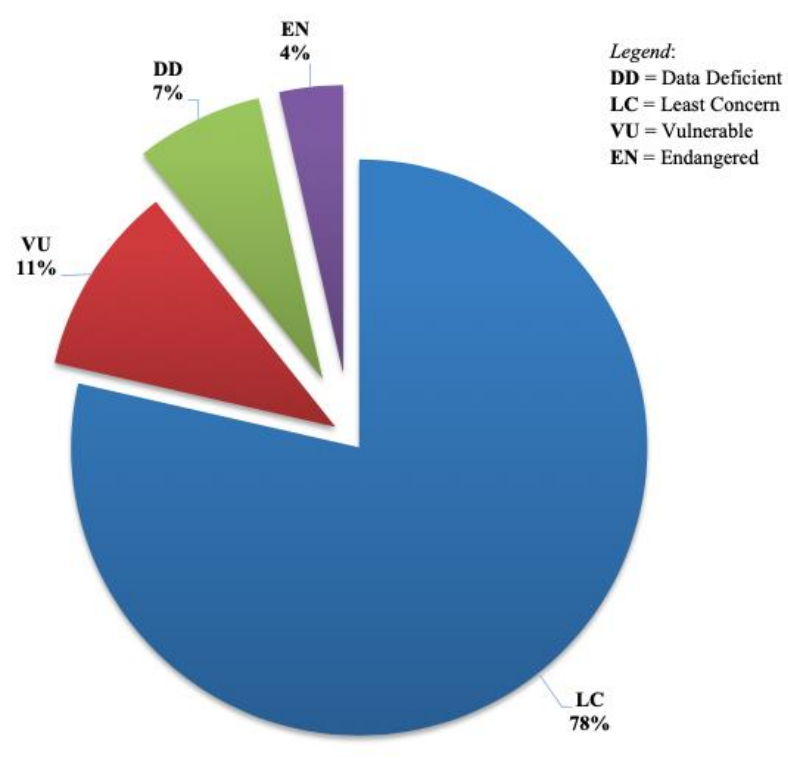

Figure 4. Conservation status of identified medicinal plants based on the International Union of Conservation of Nature (IUCN 2020).

\section{Conservation status and endemicity}

While most of the medicinal plants identified from the five barangays of Bayugan City were not assessed for their conservation status based on the international (IUCN 2019) lists of threatened species, 38 plant species were recounted in different categories (Table 1; Figure 4). Conservation status of the documented medicinal plants showed 22 species as Least Concern (LC), three species as Vulnerable

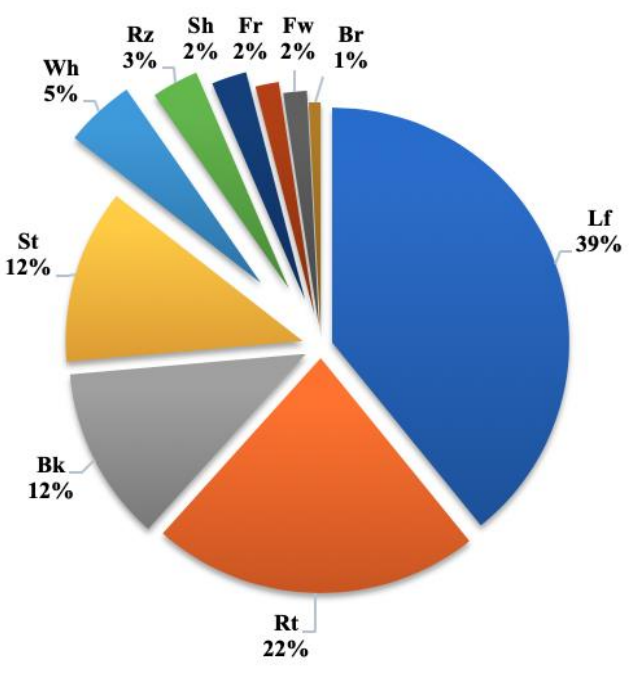

Figure 3. Plant parts used by the Agusan Manobo of Bayugan City, Philippines for medicinal application. Bk, barks; $\mathrm{Br}$, branches; Fr, fruits; Fw, flowers; Lf, leaves; Rt, roots; Rz, rhizomes; Sh, shoots; St, stems; Wh, whole plant

(VU), two species as Data Deficient (DD), and a single species as Endangered (EN), namely Swietenia mahagoni (L.) Jacq. The national list of threatened species (DENR Administrative Order 2017; CDFP; Pelser et al. 2011 onwards) revealed three species as Other Threatened Species (OTS), namely Cinnamomum mercadoi S.Vidal, Machilus philippinensis Merr., and Angiopteris evecta Sw; and a Near Threatened (NT) species, namely Calamus megaphyllus Becc.

Species distribution in terms of endemicity listed 11 species as endemic (12.6\%) while the majority of species are distributed in other countries $(87.4 \%)$, which are assumed to have been introduced or naturalized in the Philippines (CDFP; Pelser et al. 2011 onwards).

\section{Discussion}

Medicinal plants remain a valuable source of medicine in the healthcare system among the Agusan Manobo community in five upland barangays of Bayugan City, Philippines. All medicinal plants were evaluated equally among the Agusan Manobo community, and consistency of folk knowledge was observed.

The highest percentage of using leaves for treating diseases was also previously reported in earlier ethnobotanical studies in the Philippines (Abe and Ohtani 2013; Ong and Kim 2014) and some other countries (Namukobe et al. 2011; Ahmad et al. 2015; Polat et al. 2015). The Agusan Manobo sometimes prepares more than one plant parts like leaves, stems, and bark of the same species and in combination with other species for a synergistic efficiency against certain diseases.

The highest FIVs among the documented medicinal plants could be supported by some related studies of the same family. Piperaceae (pepper family) comprising ca. 
1,000 species of herbs distributed in tropical areas of India, Southeast Asia, and Africa (Scott et al. 2008) are known to have potential gastroprotective, antibacterial, antioxidant, and anticancer activities (Rekha et al. 2014). The Piper extracts are widely known, particularly in South Asian medicinal practices as an effective antibacterial and feverreducing treatment (Scott et al. 2008). These reported bioactivities of Piper species may support ethnomedicinal claims of Agusan Manobo for Piperaceae as an effective treatment for skin diseases and infections.

Lauraceae (laurel family) with more than 2,500 species are distributed within the subtropics and tropics of eastern Asia, and South and North America (Simie et al. 2004). This family was found to have potential sources for chemopreventive agents (Shen et al. 2014). Bioactivity investigation of 27 Lauraceae trees by Lin et al. (2007) revealed great potential as cancer chemoprevention agents. Al-Dhubiab (2012) validated the traditional uses of Cinnamomum burmannii (Nees \& T.Nees) Blume as treatment of diseases like nausea, flatulent dyspepsia, coughs, and chest complains in Mexico. Also, $C$. burmannii exhibited pharmacological activities like analgesic, antibacterial, antioxidant, and antitumor activities (Al-Dhubiab 2012). These findings could support the key informants' highest citation of Lauraceae for cough treatment.

Apocynaceae (dogbane family) consists of ca. 5,350 recognized species in 378 genera (Endress et al. 2018). Plant habits of this family are trees, shrubs, and sometimes herbs or vines, and their characteristic feature is usually the possession of milky sap (Wiart 2006). This family is one of the traditional medicinal families investigated as useful in treatment for boils, burns, and wounds (Patel 2014) as well as several skin problems (Alamgeer et al. 2018). These pharmacological properties could support the common uses of Apocynaceae for wound healing.

Euphorbiaceae (spurge family) consists of ca. 300 genera and ca. 7,500 species of mostly herbs and some shrubs or trees (Rahman and Akter 2013). A total of 16 species belonging to 8 genera of Euphorbiaceae were identified medicinally important aside from the economic value of the family (Ernst et al. 2015). Global medicinal uses of Euphorbia L. were reported as an effective treatment for digestive system disorders, skin ailments, respiratory complaints, inflammation, and injuries (Ernst et al. 2015). These findings could support the high citation of Euphorbiaceae among the Agusan Manobo as antipyretic medicinal plant species.

Moreover, this study showed $P$. decumanum has the highest RFC, which reveals new ethnomedicinal information as an antidote for insect and snake bites to date. Recently, $P$. decumanum was evaluated containing alkaloids, flavonoids, saponins, steroids, tannins, and fatty acids, and showed low toxicity when the stem ethanolic extract was subjected to normal lymphocytes from human blood (Dapar et al. 2020b). This species seems to be characterized distinctly as ant-associated Piper species (Dyer and Palmer 2004). A significant amount of an amide alkaloid, Piplartine, was also observed in Piper species, which is a potent anticancer agent (Raja et al. 2018).
Several bioactive compounds and essential oils of other Piper species as strong agents of antioxidant, antibacterial, antifungal, and effective treatment for insect and snake bites were comprehensively reviewed by Salehi et al. (2009) which does not include the $P$. decumanum yet. An earlier study also revealed that Piper species were cited to be the most frequently used medicinal plant species for cuts and wounds among the Agusan Manobo of Sibagat, Philippines (Dapar et al. 2020c). The molecularly confirmed $P$. aduncum folk medicinal plant of Agusan Manobo possess antimicrobial activity and active constituents against pathogenic bacteria and low cytotoxic effect, which supported its wound healing potential (Dapar and Demayo 2017; Dapar et al. 2018; Dapar et al. 2020b). The presence of reported phytochemicals may also support the Agusan Manobo use of $P$. decumanum in treatment for insect and snake bites.

Of all documented species, Anodendron borneense remains no record of ethnobotanical and pharmacological investigations. However, a new triterpene ester and other chemical constituents from the aerial parts of Anodendron paniculatum A.DC. have been isolated for the first time, which exhibited significant cytotoxic activity against human cancer cell lines (Ho et al. 2018). However, no existing studies of $A$. paniculatum as a potential cure for problems of impotence and sterility as highly cited among the respondents in this study. Hence, the present study recommends further investigation of the biological and biochemical activities of both Anodendron species.

Koriem et al. (2013) demonstrated the antihyperglycemic, antihyperlipidemic and antiapoptotic activities of Micromelum minutum, which are all related to Microminutinin coumarin ingredient of the plant seeds. Identified chemical constituents for $M$. minutum also have potential biological property containing coumarins as potent cytotoxic agents against the T-lymphoblastic leukemia cell line (Susidarti et al. 2009). This finding supports the ethnomedicinal claims among Agusan Manobo for M. minutum as a treatment for cancer.

For Arcangelisia flava, its yellow root revealed to have antimicrobial and acidity-stabilizing effects (Heryani and Nugroho 2015) while the stems demonstrated antioxidant and cytotoxic activities (Keawpradub et al. 2005). This plant has been scientifically investigated to contain several alkaloids (Verpoorte et al. 1982). The presence of its primary alkaloid, berberine, has been known to be active antimicrobial and antimalarial agents (Subeki et al. 2005). The presence of these alkaloids may support the tribal claims of $A$. flava as a treatment for ulcers.

Crude methanolic extracts of Cinnamomum mercadoi exhibited antibacterial and antioxidant activities (Fuentes et al. 2010) as well as analgesic activity (Torres et al. 2003). Other Cinnamomum species like $C$. zeylanicum Breyne and C. cassia (L.) J.Presl were reported with antimicrobial activity and antitumor property, respectively, due to the present essential oils (Sharifi-Rad et al. 2017). These results could support the ethnomedicinal uses of $C$. mercadoi by the Agusan Manobo against diarrhea. $C$. mercadoi was also recorded with the highest RFC medicinal tree used among indigenous communities in 
Esperanza, Agusan del Sur (Dapar et al. 2020d). C. mercadoi has also been reviewed for a number of local medicinal uses and phytochemical investigations as one of the interesting medicinal plants in Southeast Asia (Dapar 2020e).

Biological and biochemical investigations of all reported species under the same genus or family may imply comparable pharmacological activities in treatment against various health problems and conditions. Pharmacological investigations were recently conducted to validate medicinal plants utilized among the locals and tribal communities in Mindanao such as Gliricidia sepium (Jacq.) Kunth ex Steud. (Abdulaziz et al. 2019), Dracontomelon dao (Blanco) Merr. \& Rolfe (Dela Peña 2019), Cocos nucifera L. (Uy et al. 2019), Atuna racemosa Raf. (Nadayag et al. 2019), and Allium ampeloprasum L. (Añides et al. 2019).

The results of this study also revealed the occurrence and distribution of medicinal flora in the upland areas of the Agusan Manobo tribe in Bayugan City. Their ancestral territories are habitats of abundant medicinal plant resources that should be extensively documented and protected. These findings support the issuance of Certificate of Ancestral Domain Title (CADT) by the NCIP and DENR among indigenous communities, which serve as critical components in biodiversity preservation strategy. Indigenous protected areas program should be initiated by the LGU, which confer considerable economic and cultural benefits among the Manobo and other ICCs/IPs. The government should also reinforce the IPs right to use, access, and act as stewards of their ancestral lands. Both local people and the LGU should positively get involved in biodiversity conservation programs and strategies for sustainable protection and management of medicinal plant resources as part of the world's cultural heritage.

This study presents the rich medicinal plant knowledge of Agusan Manobo living in five upland areas of Bayugan City, Agusan del Sur. Anthropogenic activities could be observed in the upland areas such as overexploitation, poaching, logging, and deforestation, hence, a need for conservation policy and strict protection must be implemented by the local government units. It is highly recommended for the Manobo peoples' intervention to actively participate as key informants in governmental programs for conservation to sustain their cultural heritage of traditional medicine and conserve these cultural community resources. Ethnomedicinal appraisal such as this study could pave the way for further pharmacological investigations and clinical studies to validate folk medicinal uses of these plants.

\section{ACKNOWLEDGEMENTS}

We are very grateful to the Manobo community for their active participation in our documentation. We also extend our gratitude to the local government units (LGUs), provincial government administration, PENRO-LGU and NCIP-LGU of Agusan del Sur, DENR-CARAGA wildlife gratuitous permit (no. R13-2019-12), USTGS-ERC permit (protocol no. GS-2019-PN007) and NCIP-CARAGA certification. The first author would like to thank his scholarship grant from the Department of Science and Technology-Accelerated Science and Technology Human Resource Development Program-National Science Consortium (DOST-ASTHRDP-NSC) and Alexander von Humboldt Foundation as a Junior Researcher. The last author thanks the Alexander von Humboldt Foundation for a renewed research stay at the University of Bayreuth (Germany) in 2019. All authors thank the financial support of the Department of Health-Philippine Institute of Traditional and Alternative Health Care (DOH-PITAHC).

\section{REFERENCES}

Abe R, Ohtani K. 2013. An ethnobotanical study of medicinal plants and traditional therapies on Batan Island, the Philippines. J Ethnopharmacol 145: 554-565.

Abdulaziz AA, Dapar MLG, Manting MME, Torres AJ, Aranas AT, Mindo RAR, Cabrido CK, Demayo CG. 2019. Qualitative evaluation of the antimicrobial, antioxidant, and medicinally important phytochemical constituents of the ethanolic extracts of the leaves of Gliricidia sepium (Jacq.). Pharmacophore 10: 72-83.

Ahmad L, Semotiuk A, Zafar M, Ahmad M, Sultana S, Liu Q, Zada MP, Abidin SZU, Yaseen G. 2015. Ethnopharmacological documentation of medicinal plants used for hypertension among the local communities of DIR Lower, Pakistan. J Ethnopharmacol 175: 138146.

Alamgeer, Sharif A, Asif H, Younis W, Riaz H, Bukhari IA, Assiri AM. 2018. Indigenous medicinal plants of Pakistan used to treat skin diseases: a review. Chin Med 13: 52.

Alcorn J. 2010. Indigenous Peoples and Conservation. MacArthur Foundation Conservation White Paper Series.

Ali A, Badshah L, Hussain F. 2018. Ethnobotanical appraisal and conservation status of medicinal plants in Hindukush Range, District Swat, Pakistan. J Herbs Spices Med Plants 24 (4):332-355.

Al-Dhubiab BE. 2012. Pharmaceutical applications and phytochemical profile of Cinnamomum burmannii. Pharmacogn Rev 6: 125-131.

Añides JA, Dapar MLG, Aranas AT, Mindo RAR, Manting MME, Torres MAJ, Demayo CG. 2019. Phytochemical, antioxidant and antimicrobial properties of the white variety of 'Sibujing' (Allium ampeloprasum). Pharmacophore 10: 1-12.

Camacho LD, Gevaña DT, Carandang AP, Camacho SC. 2015. Indigenous knowledge and practices for the sustainable management of Ifugao forests in Cordillera. Int $\mathrm{J}$ Biodivers Sci Ecosyst Serv Manag 12 (1-2):5-13.

Carpenter KE, Springer VG. 2005. The center of marine shore fish biodiversity: the Philippine Islands. Environ Biol Fishes 72: 467-480.

CBD. 2019. Convention on Biological Diversity: Indigenous Knowledge and Biodiversity. https://en.unesco.org/links/biodiversity/cbd

CDFP; Pelser PB, Barcelona JF, Nickrent DL (eds) 2011. onwards. Co's Digital Flora of the Philippines. www.philippineplants.org

Conservation International. 2012. Biodiversity hotspots: Philippines. http://www.biodiversityhotspots.org/xp/hotspots/philippines/pages/im pacts.aspx

Dapar MLG, Demayo CG. 2017. Folk medical uses of Lunas Lunasia amara Blanco by the Manobo people, traditional healers and residents of Agusan del Sur, Philippines. Sci Int (Lahore) 29 (4):823-826.

Dapar MLG, Demayo CGD, Senarath WTPSK. 2018. Antimicrobial and cellular metabolic inhibitory properties of the ethanolic extract from the bark of 'Lunas-Bagon' (Lunasia sp.). Int J Pharm Sci Res 9: 8897. DOI: 10.13040/IJPSR.0975-8232.9(1).88-97

Dapar MLG, Alejandro GJD, Meve U, Liede-Schuman S. 2020a. Quantitative ethnopharmacological documentation and molecular confirmation of medicinal plants used by the Manobo tribe of Agusan del Sur, Philippines. J Ethnobiol Ethnomed 16: 14. DOI: 10.1186/s13002-020-00363-7

Dapar MLG, Demayo CG, Meve U, Liede-Schuman S, Alejandro GJD. 2020b. Molecular confirmation, constituents and cytotoxicity evaluation of two medicinal Piper species used by the Manobo tribe 
of Agusan del Sur. Philippines. Phytochem Lett 36: 24-31. DOI: 10.1016/j.phytol. 2020.01.017

Dapar MLG, Demayo CG, Meve U, Liede-Schuman S, Alejandro GJD. 2020c. Ethnomedicinal plants used for the treatment of cuts and wounds by the Agusan Manobo of Sibagat, Agusan del Sur Philippines. Ethnobot Res App 19: 1-18 http://dx.doi.org/10.32859/era.19.31.1-18

Dapar MLG, Alejandro GJD, Meve U, Liede-Schumann S.2020d. Ethnomedicinal importance and conservation status of medicinal trees among indigenous communities in Esperanza, Agusan del Sur, Philippines. J Complement Med Res 11(1): 5971.http://dx.doi.org/10.5455/jcmr.2020.11.01.08

Dapar MLG. 2020e. Cinnamomum mercadoi S.Vidal Lauraceae. In: Franco F. (ed) Ethnobotany of the Mountain Regions of Southeast Asia. Ethnobotany of Mountain Regions. Springer, Cham. DOI: 10.1007/978-3-030-14116-5 74-1

Dela Peña JF, Dapar MLG, Aranas AT, Mindo RAR, Cabrido CK, Torres MAJ, Manting MME, Demayo CG. 2019. Assessment of antimicrobial, antioxidant and cytotoxic properties of the ethanolic extract from Dracontomelon dao (Blanco) Merr. \& Rolfe. Pharmacophore 10: 18-29.

DENR Administrative Order. 2017. Updated national list of threatened Philippine plants and their categories (DAO 2017-11). Department of Environment and Natural Resources, the Philippines. https://server2.denr.gov.ph/uploads/rmdd/dao-2017-11.pdf

Dyer LA, Palmer AN. 2004. Piper: A Model Genus for Studies of Phytochemistry, Ecology and Evolution. Kluwer Academic Publishers, New York.

Endress ME, Meve U, Middleton DJ, Liede-Schumann S. 2018 Apocynaceae. In: Kadereit JW, Bittrich V (eds) The Families and Genera of Vascular Plants (ed Kubitzki K), Vol. XV, Flowering Plants, Eudicots, Apiales, Gentianales (excl. Rubiaceae). Springer, Heidelberg, New York.

Ernst M, Grace OM, Saslis-Lagoudakis CH, Nilsson N, Simonsen HT, Rønsted N. 2015. Global medicinal uses of Euphorbia L. (Euphorbiaceae). J Ethnopharmacol 176: 90-101. DOI: 10.1016/j.jep.2015.10.025

Fuentes RG, Diloy FN, Tan IL, Balanquit BJR. 2010. Antioxidant and antibacterial properties of crude methanolic extracts of Cinnamomum mercadoi Vidal. Phil J Nat Sci 15: 9-15.

Heryani H, Nugroho A. 2015. Study of yellow root (Arcangelisia flava Merr) as a natural food additive with antimicrobial and aciditystabilizing effects in the production process of palm sugar. Procedia Environ Sci 23: 346-350

Ho DV, Hoang HNT, Vo HQ, Nguyen HM, Raal A, Nguyen HT. 2018. A new triterpene ester and other chemical constituents from the aerial parts of Anodendron paniculatum and their cytotoxic activity. J Asian Nat Prod Res 20 (2): 188-194. DOI 10.1080/10286020.2017.1336163

ILO. 2014. Indigenous Peoples Development Programme (IPDP) https://www.ilo.org/wcmsp5/groups/public/---asia/---ro-bangkok/--ilo-manila/documents/publication/wcms_245610.pdf

IPBES. 2019. Summary for policymakers of the global assessment report on biodiversity and ecosystem services of the Intergovernmental Science-Policy Platform on Biodiversity and Ecosystem Services.https://www.ipbes.net/sites/default/files/downloads/spm_une dited_advance_for_posting_htn.pdf

IPNI. 2019. The International Plant Names Index. https://www.ipni.org

IUCN. 2019.The International Union for Conservation of Nature's Red List of Threatened Species. Version 2019-2. https://www.iucnredlist.org

Keawpradub N, Dej-adisai S, Yuenyongsawad S. 2005. Antioxidant and cytotoxic activities of Thai medicinal plants named Khaminkhruea: Arcangelisia flava, Coscinium blumeanum and Fibraurea tinctoria. Songklanakarin J Sci Technol 27: 455-467.

Koriem KMM, Aminuddin ME, Kader AS, Sheikh NR. 2013. Antihyperglycemic, antihyperlipidemic and antiapoptotic activities of Micromelum minute seeds in diabetic rats. J Mol Genet Med 1: 1-8. DOI: 10.4172/1747-0862.S1-004

Lin CT, Chu FH, Tseng YH, Tsai JB, Chang ST, Wang SY. 2007. Bioactivity investigation of Lauraceaetrees grown in Taiwan. Pharm Biol 45: 638-644.

Madulid DA. 2001. A Dictionary of Philippines Plant Names. Vol. I Local Name-Scientific Name. Vol. II: Scientific Name-Local Name. Bookmark, Makati City.
Myers N, Mittermeier RA, Mittermeier CG, da Fonseca GAB, Kent J. 2000. Biodiversity hotspots for conservation priorities. Nature 403: 853-858.

Nadayag J, Dapar MLG, Aranas AT, Mindo RAR, Cabrido CK, Manting MME, Torres AJ, Demayo CG. 2019. Qualitative assessment of the antimicrobial, antioxidant, and phytochemical properties of the ethanolic extracts of the inner bark of Atuna racemosa. Pharmacophore 10: 52-59.

Namukobe J, Kasenene JM, Kiremire BT, Byamukama R, KamatenesiMugisha M, Krief S, Dumontet V, Kabasa JD. 2011. Traditional plants used for medicinal purposes by local communities around the Northern sector of Kibale National Park, Uganda. J Ethnopharmacol 136: $236-245$

NCCA. 2015. Manobo. http://ncca.gov.ph/about-culture-and-arts/cultureprofile/manobo/

NCIP. 2010. Primer on Census for Indigenous Peoples. National Commission on Indigenous Peoples, Quezon City, Philippines.

Ong HG, Kim YD. 2014. Quantitative ethnobotanical study of the medicinal plants used by the Ati Negrito indigenous group in Guimaras Island, Philippines. J Ethnopharmacol 157: 228-242.

Patel DK. 2014. Some traditional medicinal plants useful for boil burn and for wounds healing. J Biodivers Endanger Species 2: 133.

PENRO Agusan del Sur. 2019. Provincial Environment and Natural Resources Office-Agusan del Sur Land Classification. http://www.denrpenroads.com/index.php/about/background

Polat R, Cakilcioglu U, Kaltalioglu K, Ulusan MD, Türkmen Z. 2015. An ethnobotanical study on medicinal plants in Espiye and its surrounding (Giresun-Turkey). J Ethnopharmacol 163: 1-11.

Posa MRC, Diesmos AC, Sodhi NS, Brooks TM. 2008. Hope for threatened tropical biodiversity: lessons from the Philippines. Bio Sci 58: $231-240$.

PSA. 2016. 2010 Census of Population and Housing: Definition of Terms and Concepts. Philippine Statistics Authority, Quezon City, Philippines.

Rahman AHM, Akter M. 2013. Taxonomy and medicinal uses of Euphorbiaceae (Spurge) family of Rajshahi, Bangladesh. Res Plant Sci 1: 74-80.

Raja MR, Rukayadi Y, Maulidiani M, Ismail IS. 2018. Solvent extraction and identification of active anticariogenic metabolites in Piper cubeba L. through ${ }^{1} \mathrm{H}-\mathrm{NMR}$-Based metabolomics approach. Molecules 23: 1730. DOI: $10.3390 /$ molecules 23071730

Rekha VP, Kollipara M, Gupta BRSS, Bharath Y, Pulicherla K. 2014. A review on Piper betle L.: nature's promising medicinal reservoir. Am J Ethnomed 1: 276-289.

ReyesCM, Mina CD, Asis RD. 2017. Inequality of opportunities among ethnic groups in the Philippines. United Nations University World Institute for Development Economic Research. WIDER Working Paper 154.

Reyes-García V, Benyei P. 2019. Indigenous knowledge for conservation. Nat Sustain 2: 657-658.

Salehi B, Zakaria ZA, Gyawali R, Ibrahim SA, Rajkovic J, Shinwari ZK, Khan T, Sharifi-Rad J, Ozleyen A, Turkdonmez E, Valussi M, Tumer TB, Fidalgo LM, Martorell M, Setzer WN. 2009. Piper Species: a comprehensive review on their phytochemistry, biological activities and applications. Molecules 24 (7): 1364 DOI: $10.3390 /$ molecules 24071364

Scott IM, Jensen HR, Philogène BJR, Arnason JT. 2008. A review of Piper spp. (Piperaceae) phytochemistry, insecticidal activity and mode of action. Phytochem Rev 7: 65-75.

SDG. 2019. Sustainable Development Goals: UN report: nature's dangerous decline 'unprecedented'; species extinction rates' accelerating'.

https://www.un.org/sustainabledevelopment/blog/2019/05/naturedecline-unprecedented-report/

Sharifi-Rad J, Sureda A, Tenore GC, Daglia M, Sharifi-Rad M, Valussi M, Tundis R, Sharifi-Rad M, Loizzo MR, Ademiluyi AO, Sharifi-Rad R, Ayatollahi SA, Marcello I. 2017. Biological activities of essential oils: from plant chemoecology to traditional healing systems. Molecules 22:70

Shen T, Chen XM, Harder B, Long M, Wang XN, Lou HX, Wondrak GT, Ren DM, Zhang DD. 2014. Plant extracts of the family Lauraceae: a potential resource for chemopreventive agents that activate the nuclear factor erythroid 2-related factor 2/antioxidant response element pathway. Planta Med 80: 426-434. 
Simie A, Sokovie MD, Ristic M, Grujic-Jovanovic S, Vukojevic J, Marin PD. 2004. The chemical composition of some Lauraceae essential oils and their antifungal activities. Phytother Res 18: 713-717.

Subeki, Nomura S, Matsuura H, Yamasaki M, Yamato O, Maede Y, Katakura K, Suzuki M, Trimurningsih C, Yoshihara T. 2005. Antibabesial activity of some Central Kalimantan plant extracts and active oligostilbenoids from Shorea balangeran. Planta Med 71: 420-423.

Susidarti RA, Rahmani M, Ismail HBM, Sukari MA, Hin TYY, Lian GEC, Ali AM. 2009. Cytotoxic activity of coumarins from Micromelum minutum. Pharm Biol 47: 182-185. DOI: $10.1080 / 13880200802436513$

The Plant List. 2013. Version 1.1. http://www.theplantlist.org/

Torres RC, Sison FM, Ysrael MC. 2003. Phytochemical screening and biological studies on the crude methanol extract of Cinnamomum mercadoi Vidal. Philipp J Sci 132: 27-32.

Tropicos. 2019. Missouri Botanical Garden. http://www.tropicos.org

Ugulu I, Baslar S, Yorek N, Dogan Y. 2009. The investigation and quantitative ethnobotanical evaluation of medicinal plants used around Izmir Province, Turkey. J Med Plant Res 3: 345-367.

UNDP. 2010. Indigenous Peoples in The Philippines. https://www.undp.org/content/dam/philippines/docs/Governance/fast
Facts6\%20-

$\% 20$ Indigenous $\% 20$ Peoples $\% 20$ in $\% 20$ the $\% 20$ Philippines $\% 20$ rev $\% 2$ 01.5.pdf

UNESCO. 2019. Indigenous knowledge and biodiversity. https://en.unesco.org/links/biodiversity

Uy IA, Dapar MLG, Aranas AT, Mindo RAR, Manting MME, Torres MAJ, Demayo CG. 2019. Qualitative assessment of the antimicrobial, antioxidant, phytochemical properties of the ethanolic extracts of the roots of Cocos nucifera L. Pharmacophore 10: 63-75.

Verpoorte R, Siwon J, Essen GFA, TiekenM, SvendsenAB. 1982. Studies on Indonesian medicinal plants: alkaloid of Arcangelisia flava. J Nat Prod 45: 582-584.

Vinyeta K, Lynn K. 2013. Exploring the role of traditional ecological knowledge in climate change initiatives. Gen Tech Rep PNW-GTR879. U.S. Department of Agriculture, Forest Service, Pacific Northwest Research Station, Portland.

Wiart C. 2006. Medicinal Plants of Asia and The Pacific. CRC Press/Taylor and Francis, Boca Raton.

WRI. 2005. Securing Property and Resource Rights through Tenure Reform. World Resources Report 2005: The Wealth of the Poor Managing Ecosystems to Fight Poverty. Washington, D.C. 